\title{
A Smart-hand Movement-based System to Control a Wheelchair Wirelessly
}

\author{
Fahd N. Al-Wesabi, ${ }^{1,3^{*}}$ Mohammad Alamgeer, ${ }^{2}$ \\ Fuad Al-Yarimi, ${ }^{2}$ and Adnan Albaadani ${ }^{3}$ \\ ${ }^{1}$ Department of Computer Science, King Khalid University, Muhayel Aseer, KSA \\ ${ }^{2}$ Department of Information Systems, King Khalid University, Muhayel Aseer, KSA \\ ${ }^{3}$ Faculty of Computer and IT, Sana'a University, Sana'a, Yemen
}

(Received June 12, 2019; accepted August 5, 2019)

Keywords: microcontroller, accumulator, partially quadriplegic, hand gestures

The number of elderly and disabled people worldwide has increased and their day-to-day activities depend on others' help. Improving the quality of life of these people has become the most important responsibility of society and it is the role of technology specialists to make their life as normal as possible and easy so that they can do their day-to-day activities at the right time without others' help. Many researchers have proposed several solutions, but they have limitations such as poor performance and usability. In this paper, we propose a smart wirelessbased wheelchair system that completely controls the motion of a wheelchair wirelessly through hand movements to help partially quadriplegic people to perform their daily activities easily. In the proposed approach, sensors with relevant materials and technologies have been integrated with microcontrollers to capture hand movement signals and process them to the fully control the wheelchair wirelessly. An accumulator sensor is placed on the user's hand to acquire the directions of hand movements and translate them into movement commands using an Arduino microcontroller that is directly connected to the wheelchair and moves it. The proposed system has been simulated, and the obtained results show the effectiveness of the proposed system and its applicability for use by most physically disabled persons.

\section{Introduction}

Wheelchair users are the most visible among the physically disabled community. Elderly and partially quadriplegic people are the group with the highest rates of manual and electric wheelchair use. ${ }^{(1)}$ Wheelchair users face difficulties in their daily activities. It is difficult for the elderly and partially quadriplegic people to maneuver electrical and mechanical wheelchairs.

Nowadays, a wide range of technologies is available to help the disabled and physically challenged, and many things around us heavily depend on technologies that make life easier and more flexible. ${ }^{(1)}$ These technologies gave solutions to problems that are difficult to solve by humans. ${ }^{(2,3)}$ Control systems are the most recent technological advancement designed for

*Corresponding author: e-mail: falwesabi@kku.edu.sa https://doi.org/10.18494/SAM.2019.2468 
various purposes, specifically to help the disabled and physically challenged, and to replace conventional manual support systems. ${ }^{(4,5)}$

According to the results of a recently completed clinical trial, an assistive technology that enables individuals to maneuver a powered wheelchair with a variety of guided systems, such as those using a mouse cursor or a joystick and a tactile screen, and systems based on voice recognition, can be operated by individuals with a certain amount of upper body mobility. ${ }^{(2,10)}$ Moreover, technologies provide solutions in the medical field, which is our concern in this paper. Therefore, many attempts have been made to develop various solutions to help those with disabilities to move and to minimize their level of dependence on others to help them move. ${ }^{(6)}$

Elderly and partially quadriplegic people suffering from severe paralysis may not be able to use these technologies since they require accurate control. To improve the lifestyle of the physically challenged, in this work, we aim to develop a wheelchair system that moves in accordance with the signals obtained from hand movements through an accumulator sensor. Since hand movements are limited and identified by processing data, in this work, we aim also to explore the signals collected by the accumulator for better maneuver ability of the wheelchair.

The accumulator sensor is used to acquire specific activities and convert them to an analog signal by using encoder and decoder protocols, and send them to other connected devices by using an RF transmitter and an RF receiver. A microcontroller (AT89C51) is used to analyze the encoded signals and translate them into useful commands. An Arduino microcontroller (ATMEGA328P-PU) is used to directly connect the devices in order to perform fully wired or wireless control.

In this study, using the above-mentioned techniques, we developed a smart wireless wheelchair control using hand gesture (SWWCHG) system to solve physical disability challenges and assist the elderly and fully or partially quadriplegic people to perform their daily life activities without others' help. The SWWCHG system consists of a model that performs in collaboration with an accumulator sensor with relevant materials, technologies, and an Arduino microcontroller unit, which is directly connected to the wheelchair and moves it. The hand movements of people are used to achieve full control of a wireless wheelchair.

The major objective of the SWWCHG system is to solve physical disability challenges and improve the quality of life of the elderly and fully or partially quadriplegic people, and help them perform their daily life activities without others' help.

The main contributions of our proposed system are as follows.

1) This new SWWCHG system will improve the day-to-day life quality of the elderly and partially or fully quadriplegic people.

2) It provides a solution familiar to users with full wireless control of a wheelchair using hand movements.

3) Hand gestures are identified and analyzed using a microcontroller without the need for a computer to control the wheelchair.

To the best of our knowledge, the SWWCHG system, which adopts hybrid techniques, is efficient. Baseline approaches make use of traditional wired techniques that are not familiar to elderly and partially or fully quadriplegic people. However, the SWWCHG system is wireless 
and a hybrid because it uses both the above-mentioned mechanisms and addresses the problems encountered in using and controlling a wheelchair by elderly and disabled users. The rest of the paper is organized as follows. In Sect. 2 we explain the existing work done so far. In Sect. 3 , we present the proposed system. In Sect. 4, we describe the simulation and implementation procedures. In Sect. 5, we provide the results and discussion. Finally, in Sect. 6, we conclude this paper.

\section{Literature Review}

Over the years, several approaches, such as joystick, gesture and chain-based control mechanisms, have been proposed to help physically disabled people reduce their level of dependence on others for movement. An image-processing-based approach has been proposed in Ref. 3 to control a wheelchair. This approach relies on image processing to recognize a gesture. The proposed approach is easy to handle and operate by users. However, it requires a wired web camera to perform the operation, which makes it unreliable and difficult to use. A gesture-based wheelchair approach has been proposed in Ref. 4 to control a wheelchair using hand movements for disabled people. The proposed approach uses the MEMS sensor connected to the hand and 3 -axis accelerometer with digital output $\left(12^{\mathrm{C}}\right)$, which detects and converts hand gestures to the 6-bit digital values. The main drawback of this approach using a remotely controlled system, is that the manner of holding the remote control may inconvenience users.

A smart wheelchair prototype based on hand gesture control has been proposed in Ref. 5 to help disabled people control a wheelchair. Many wired hardware components are required in this prototype, which makes it inconvenient for users. A model for a hand-gesture-controlled user interface has been presented in Ref. 6 to control a wheelchair using hand movements by using an accelerometer sensor. The main drawback of this model is its low accuracy, which makes the model inapplicable for users with low confidence. In addition, wired devices such as a global system for a mobile communications (GSM) modem are required, making the use of the model more difficult for the elderly and physically disabled people.

A hand-gesture-based approach using a touch sensor for users to control a wheelchair effectively has been proposed in Ref. 7. This approach however uses a wired hand gesture hardware component, which makes it difficult for the elderly and physically disabled people to use. In Ref. 8, the authors proposed a hand-gesture-based approach to control a wheelchair. This approach relies on the use of the global positioning system (GPS) and GSM to identify locations, which makes it unreliable to use in noisy environments.

The hand-gesture-based approach that uses Raspberry pi and image processing techniques to control a wheelchair has been proposed in Ref. 9. This approach relies on an image processing technique and a USB web camera to recognize gestures. The main drawback of this approach is that it is difficult to handle and operate by users, and it requires a wired web camera to perform the operations. An accelerometer-based-gesture approach has been proposed in Ref. 11 to control a wheelchair using GPS and GSM navigation. In this approach, a wheelchair component should be transmitted to a control room and the location of the user is determined by a navigation application. In Ref. 12, the authors presented a framework to help people who 
cannot walk owing to physiological or physical illness. The proposed framework involves the use of computer-controlled wheelchairs, which will be unfamiliar to the elderly and physically disabled people.

A model for a hand-gesture-wired-controlled user interface has been presented in Ref. 13; this model uses an accelerometer sensor to control the direction of a wheelchair using hand movements. The main drawback of the proposed model is that the transmission control is wired, making it difficult for users to handle and operate. In Refs. 14-16, 18, and 25, the authors developed hand-gesture-based methods to control wheelchair movement using MEMS and acceleration technologies. The main drawbacks of these methods are their non-costeffectiveness and difficulty to use and operate because they are unfamiliar to the elderly and severely physically disabled people. A gesture-recognition-based approach has been proposed in Ref. 17 to control a wheelchair using an android application. A smartphone and a connection should be used to control a wheelchair. Using the touch screen of the smartphone, the user has to choose the direction specified within the four quadrants on the screen, which is difficult for users to use. In Ref. 19, the authors present a hand-gesture-reorganization approach using a real-time tracking method and a hidden Markov model that introduces the hand gesture reorganization system to recognize continuous gestures before a stationary background. In this approach, the motion of the object gives important and useful information for object localization and extraction. However, to recognize the gesture, the complexity is higher and the accuracy is lower, so it is not beneficial and not compatible with the users.

A visual-based human-machine interface (HMI) solution has been proposed in Ref. 20 to control a wheelchair by head gestures, which were recognized by detecting the position of the nose on a user's face. The proposed approach is uncomfortable and not applicable to all users, which requires constant shoulder or neck movements. The head of the user should always be within the range of the sensor; otherwise, the user cannot control the movement of the cursor. In Ref. 21, the authors presented an approach using closeness matching to detect hand motion, whereas in Ref. 22, the authors presented a method that utilize a strategy for signal coordination with the utilization of the ARM 11 Raspberry pi and Zigbee module. Another approach that uses wearable hand gloves to capture hand movement to control a wheelchair has been proposed in Ref. 23. In Ref. 24, the authors described their work on gesture reorganization applied to wheelchair control. In this case, gestures are recognized through a three-axis accelerometer sensor.

\section{Proposed System}

In this paper, we propose the SWWCHG system to overcome physical disability challenges and assist the elderly and fully or partially quadriplegic people to perform their daily life activities without others' help.

We assume the following for our system:

1) An accumulator is placed on the user's hand to sense the movements of the hand and change them into analog signals; then, the signals will be directed to an encoder to be sent by an RF transmitter. 
2) The hand movements of the user will be translated into movement commands by an Arduino microcontroller unit that is directly connected to the wheelchair to move it.

3) The hand movements of the user will be converted into numeric data using transmission and reception circuits.

4) There are five commands that provide adequate and proper control, namely, forward, backward, turn right, turn left, and stop commands.

\subsection{Core components of SWWCHG}

In the SWWCHG system, an accumulator with an RF transmitter, an RF receiver, and microcontrollers (AT89C51 and ATMEGA328P-PU) are used as its core components. These components are integrated with a wheelchair and programmed to fully control the movement and directions of the wheelchair. Figure 1 shows the core components and work mechanism of the SWWCHG system.

\subsubsection{Hand gestures}

For people who are partially paralyzed and can move their hands only straightly, we provide a solution that depends on hand gestures to represent the directions of a wheelchair, as shown in Fig. 2.

\subsubsection{Accumulator}

The accumulator in the proposed system is used to sense the movements of the hand and change them into analog signals, which will then be directed to an encoder sent by an RF transmitter, as shown in Fig. 3.

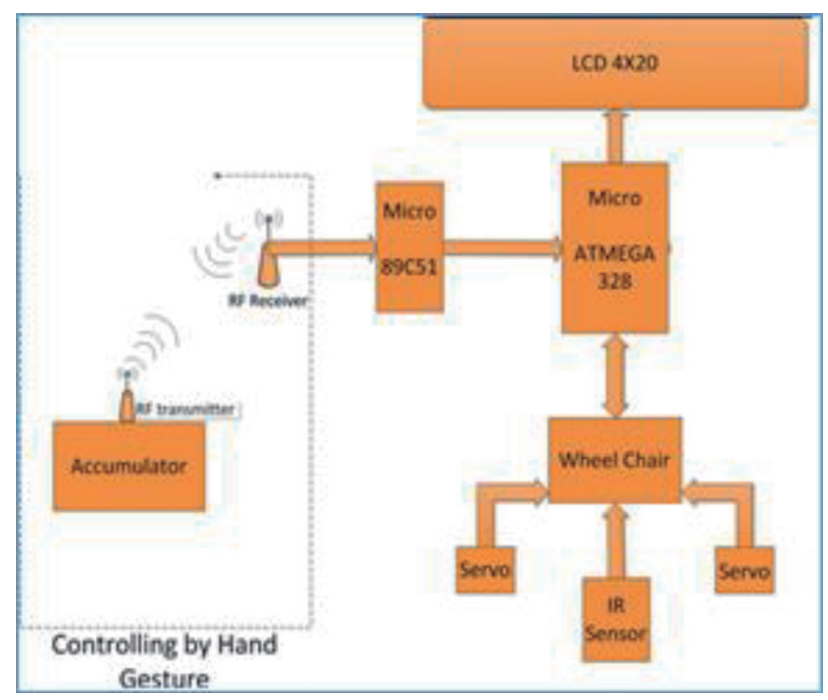

Fig. 1. (Color online) Core components of the SWWCHG system. 

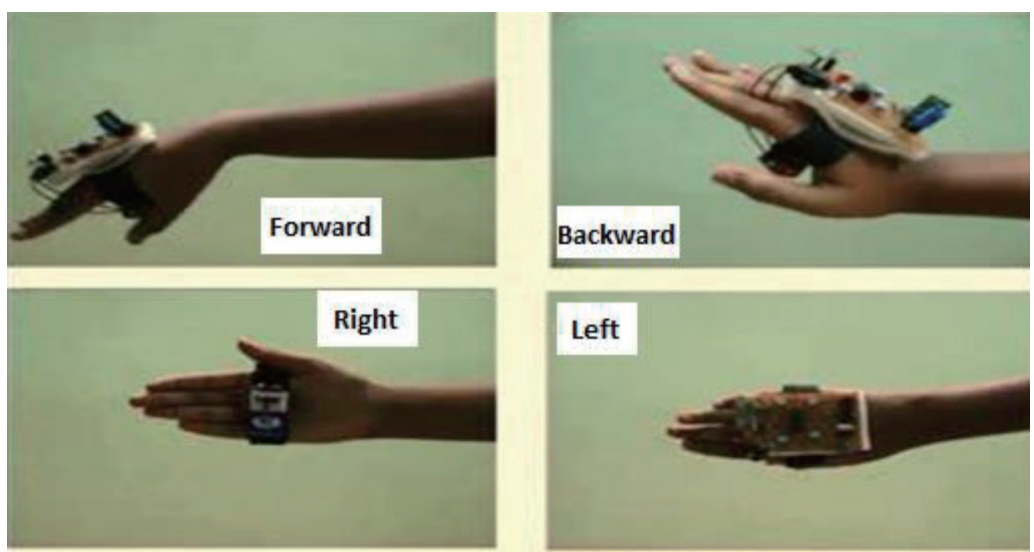

Fig. 2. (Color online) Hand movements and the commands they represent when using the SWWCHG system.

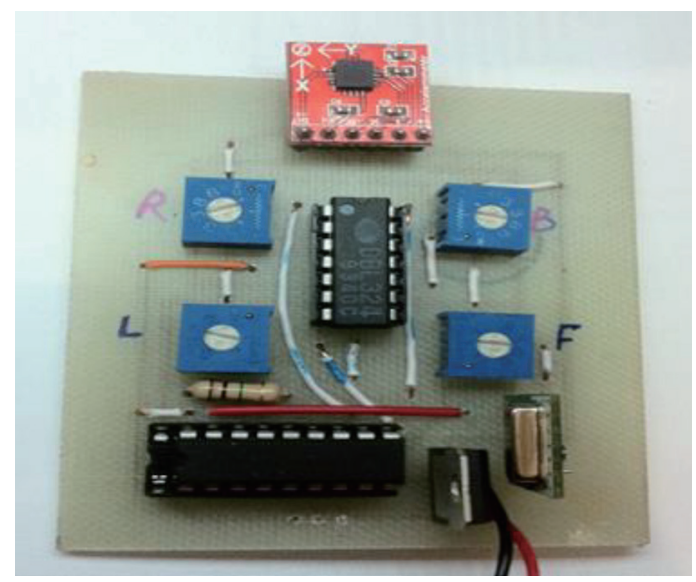

Fig. 3. (Color online) Accumulator on the board of the SWWCHG system.

\subsubsection{AT89C51 microcontroller}

After the signals are received by the RF receiver to be directed to the encoder (to be encoded), we use another microcontroller (AT89C51) to analyze the encoded signals and then send them to the ATMEGA328P-PU microcontroller to convert them into movement commands, as shown in Fig. 4.

\subsubsection{Servo motor, IS sensor, and wheelchair}

A wheelchair is directly connected to the ATMEGA328P-PU microcontroller. Small controlled servo motors are also connected to the wheelchair for speed control. An IS sensor is connected to the wheelchair to measure distance and stop the servo motors from moving if there is a barrier in front of the wheelchair. 


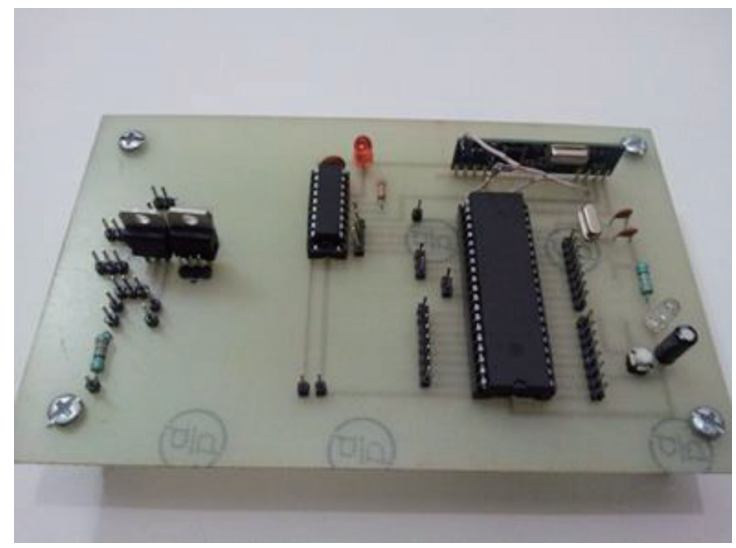

Fig. 4. (Color online) AT89C51 microcontroller on the board of the SWWCHG system.

\subsection{Conversion of hand movements into numeric data}

The proposed system uses two circuits for the transmission and reception of data. The work mechanism of each will be explained separately as follows.

\subsubsection{Transmission circuit}

This circuit will be installed on a user's hand to identify hand movements, which are forward, backward, rightward, and leftward. After identifying the hand movements that can be read, we assigned each movement to each of the five commands as follows:

- The order to move forward corresponds to the forward movement of the hand.

- The order to move backward corresponds to the backward hand movement.

- A right turn corresponds to the hand movement to the right.

- A left turn corresponds to the hand movement to the left.

- The stop command corresponds to the hand remaining straight without movement toward any direction.

The direction of the hand movement depends on the output of the motion sensor, which gives various efforts when moving the hand. $X$ - and $Y$-axes were used only to determine the direction of the hand movement. The hand movement to the front and back is represented by the $X$-axis, and that to the right and left is represented by the $Y$-axis, as shown in Fig. 2.

The following mechanism has been used to determine the directions of the hand movements and send them through an RF transmitter:

1) The direction of the hand movement is determined by using the accumulator. Since the accumulator gives analog output, it should be converted into digital output using the LM324 IC comparator. The accumulator output is compared with a reference voltage, and this voltage is approximated to half the sensitivity of the input voltage. An experiment showed that the appropriate reference voltage for the circuit is $2.55 \mathrm{~V}$, because the accumulator 
output is higher or lower than this value when a movement occurs. The comparator gives either a high- or low-voltage output through the ports $(1,7,8,14)$ to provide digital output in four bits. Table 1 shows the relationship between the directions of hand movements and the comparator output.

2) The HT12E encoder is used to convert the four bits from parallel processing to serial data so that the data can be transmitted via the transmitter.

3) The outputs of the encoder will be the inputs to the RF transmitter to send them to the receiving circuit.

Figure 5 shows a diagram of the transmission circuit.

\subsubsection{Reception circuit}

This circuit receives the data transmitted by the transmission circuit and modifies them using the AT89c51 controller, and then sends them to the ATMega328P-PU controller to control the wheelchair. The mechanism used to perform these tasks is as follows:

a. The data transmitted is received by the $\mathrm{RF}$ receiver $(433 \mathrm{MHz})$.

b. The data is serialized when received and therefore passed through the HT12D decoder in parallel as four-bit output by ports $(10,11,12$, and 13).

c. The data is sent to the AT89c51 controller to be processed and represented according to the hand movement in three-bit form, and then sent to the three remaining ports of the ATMega328P-PU controller to convert them into the control commands of the wheelchair. Figure 6 shows the diagram of the reception circuit.

Table 1

Relationship between directions of the hand movements and comparator output.

\begin{tabular}{lcccccccccccccccc}
\hline $\begin{array}{l}\text { Direction of hand } \\
\text { movement }\end{array}$ & \multicolumn{3}{c}{ Forward } & \multicolumn{3}{c}{ Backward } & \multicolumn{3}{c}{ Rightward } & \multicolumn{3}{c}{ Leftward } \\
\hline Port numbers & 1 & 7 & 8 & 14 & 1 & 7 & 8 & 14 & 1 & 7 & 8 & 14 & 1 & 7 & 8 & 14 \\
Port output values & 1 & 0 & 0 & 0 & 0 & 1 & 0 & 0 & 0 & 0 & 1 & 0 & 0 & 0 & 0 & 1 \\
\hline
\end{tabular}

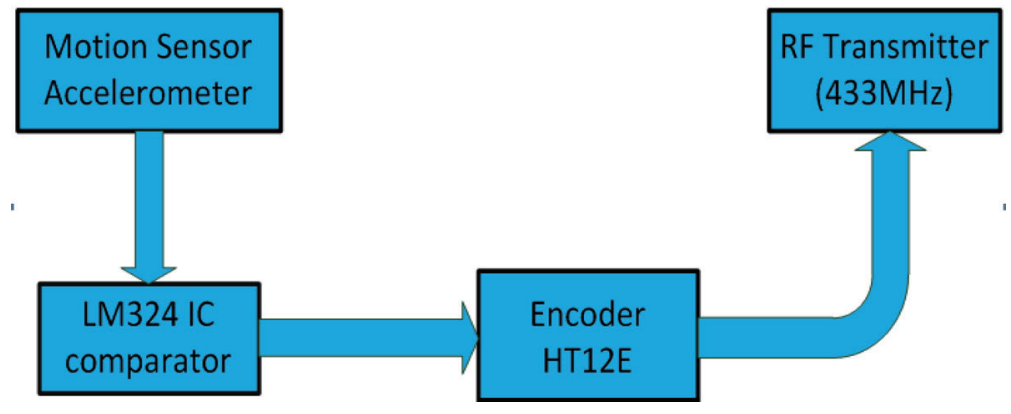

Fig. 5. (Color online) Diagram of the transmission circuit of the SWWCHG system. 


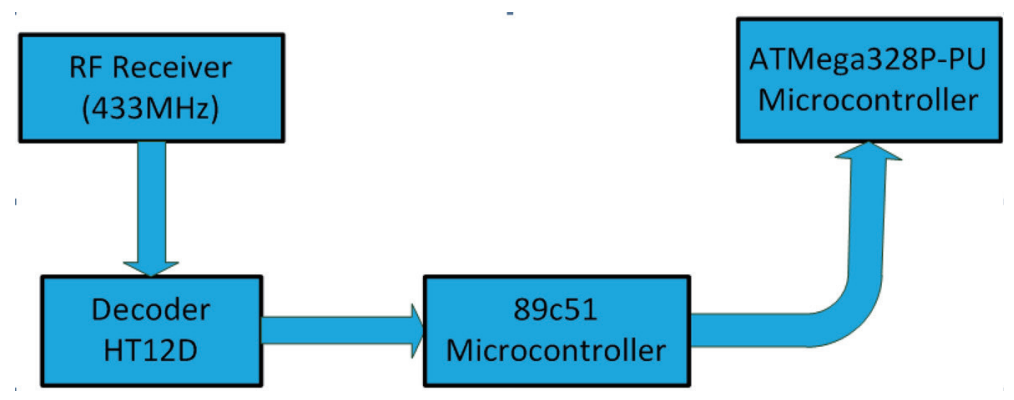

Fig. 6. (Color online) Diagram of the reception circuit of the SWWCHG system.

\subsection{Convert hand movements to control commands of servo motors}

Here, we will explain how to connect the ATMega328P-PU controller to the AT89c51 controller and convert hand movements to commands to control the servo motors of a wheelchair.

There are three ports available in the ATMega328P-PU controller. Since there are five hand movements, these movements can be expressed using only three bits; thus, the four-bit data should be converted to three-bit data. The following steps have been taken to convert the data from four to three bits as well as to convert hand movements into commands:

1) The AT89c51 controller has been programmed using $C$ programming language, which will read the data from the decoder12D and convert it from four to three bits, using the program Keil uVision4.

2) The three ports (P2.2, P2.1, and P2.0) of the AT89c51 controller are connected to the ports $(18,19$, and 27) of the ATMega328P-PU controller, and these ports will be used to read data on the hand movements and convert them into commands. These ports in the Arduino are called digital pin13, digital pin12, and analog pin4. Figure 7 shows the graph of the data conversion algorithm to three bits as shown in Table 2.

Figure 7 shows the flowchart of the receiving circuit to convert the data into three bits.

3) An appropriate algorithm has been developed and implemented using Arduino $\mathrm{C}$ for the ATMega328P-PU controller to convert the hand movement data received by the AT89c51 controller to the control commands of the wheelchair. Table 2 shows how to convert hand movements into commands to control the wheelchair and convert them to 3-bit data.

Figure 8 shows the flowchart of the hand-translation algorithm for commands to control the wheelchair.

\section{Simulation, Implementation, and Discussion}

To evaluate the performance and efficiency of our proposed system, we carried out simulation and implementation using the $\mathrm{C}$ programming language with an Arduino $\mathrm{C}$ IDE and Protues simulation software. The IDE has several built-in functions and falls under the AVRembedded $\mathrm{C}$ design based on processing. 


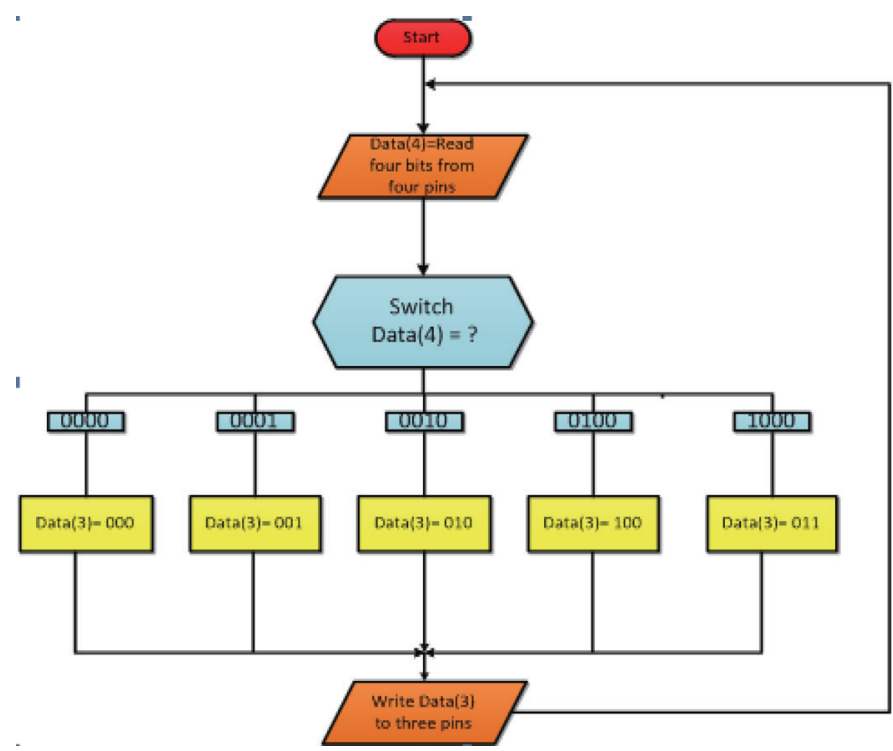

Fig. 7. (Color online) Flowchart to convert 4-bit data into 3-bit data using SWWCHG system.

Table 2

Conversion of hand movements into commands to control motor movement and data conversion to 3 bits.

\begin{tabular}{|c|c|c|c|c|}
\hline $\begin{array}{l}\text { Direction of hand } \\
\text { movement }\end{array}$ & Motor movement & $\begin{array}{l}\text { Control wheelchair } \\
\text { model movement }\end{array}$ & 4-bit data & 3-bit data \\
\hline Forward & $\begin{array}{l}\text { Rotate the two motors } \\
\text { forward }\end{array}$ & Move forward & 0001 & 001 \\
\hline Backward & $\begin{array}{c}\text { Rotate the two motors } \\
\text { backward }\end{array}$ & Move backward & 0010 & 010 \\
\hline Rightward & $\begin{array}{l}\text { Rotate the left motor } \\
\text { to the front }\end{array}$ & Turn right & 0100 & 100 \\
\hline Leftward & $\begin{array}{l}\text { Rotate the right motor } \\
\text { to the front }\end{array}$ & Turn left & 1000 & 011 \\
\hline No hand movement & $\begin{array}{l}\text { Stop the spinning } \\
\text { of two motors }\end{array}$ & $\begin{array}{l}\text { Wheelchair stops } \\
\text { moving }\end{array}$ & 0000 & 000 \\
\hline
\end{tabular}

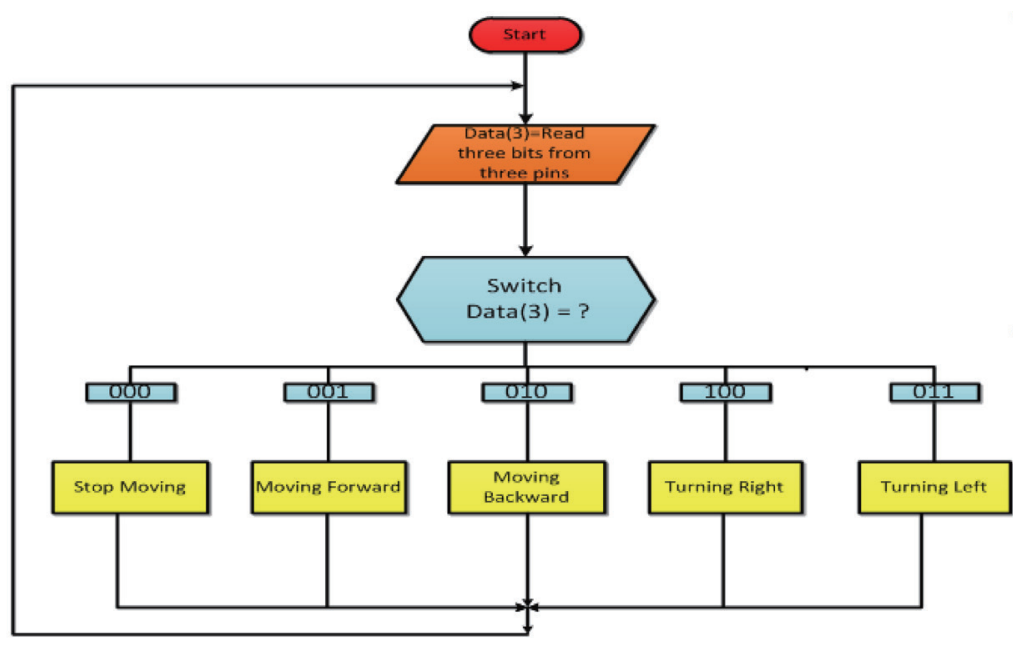

Fig. 8. (Color online) Flowchart of the wheelchair model control by hand movement using SWWCHG system. 


\subsection{Accumulator simulation}

The accumulator was tested in four cases in a practical experiment on the sensitivity of the sensor at a $45^{\circ}$ angle. When the feeding voltage was $5 \mathrm{~V}$, the following values were given as shown in Table 3.

From Table 3, we show the following observations:

- The $X, Y$, and $Z$ values at the fixed level are found in the range of 2.48-2.68.

- If the sensor is tilted forward, the $X$ value increases; however, if it is tilted backward, the $X$ value decreases.

- If the sensor is tilted to the left, the $Y$ value increases; however, if it is tilted to the right, the $Y$ value decreases.

- The $Z$ values is almost constant in previous cases.

\subsection{Simulation of connecting an accumulator with a voltage comparator}

The $X$ and $Y$ outputs from the sensor ports are connected to the $1 \mathrm{~m} 324$ voltage comparator, where the obtained values are compared to read the change; there are four voltage comparisons, each of which is linked as follows:

- 1st comparison: The increase in $X$ value is compared with the constant level by a reference voltage; this value is input via the inverting port of the comparator.

- 2nd comparison: The decrease in $X$ value is compared with the constant level by a reference voltage; this value enters via the nonaveraging negative port of the comparator.

- 3rd comparison: The increase in $Y$ value is compared with the constant level by a reference voltage; this value is input via the inverting positive port of the comparator.

- 4th comparison: The decrease in $Y$ value is compared with the constant level by a reference voltage; this value enters via the noninverting negative port of the comparator.

A variable voltage resistor is connected to the variable voltage to adjust the reference voltage for comparison. Figure 9 shows the simulated link of the accumulator with the voltage comparator.

\subsection{Encoder and decoder connection}

The output data from the $1 \mathrm{~m} 324$ voltage comparator connects to this unit in parallel. Output should be sequentially so that it will be easy to send wirelessly and the input ports are arranged as front, back, right, and left and output through one serial port. On the decoder side, the data enters this unit through the wireless receiver as serial data and outputs it in parallel.

Table 3

Readings of motion sensor values.

\begin{tabular}{lccc}
\hline Accumulator direction & $X$ values & $Y$ values & $Z$ values \\
\hline Front & $2.79-2.80$ & 2.55 & $2.99-3.00$ \\
Back & 2.31 & 2.59 & 2.96 \\
Right & 2.55 & $2.43-2.44$ & 2.99 \\
Left & 2.56 & 2.72 & 2.97 \\
\hline
\end{tabular}




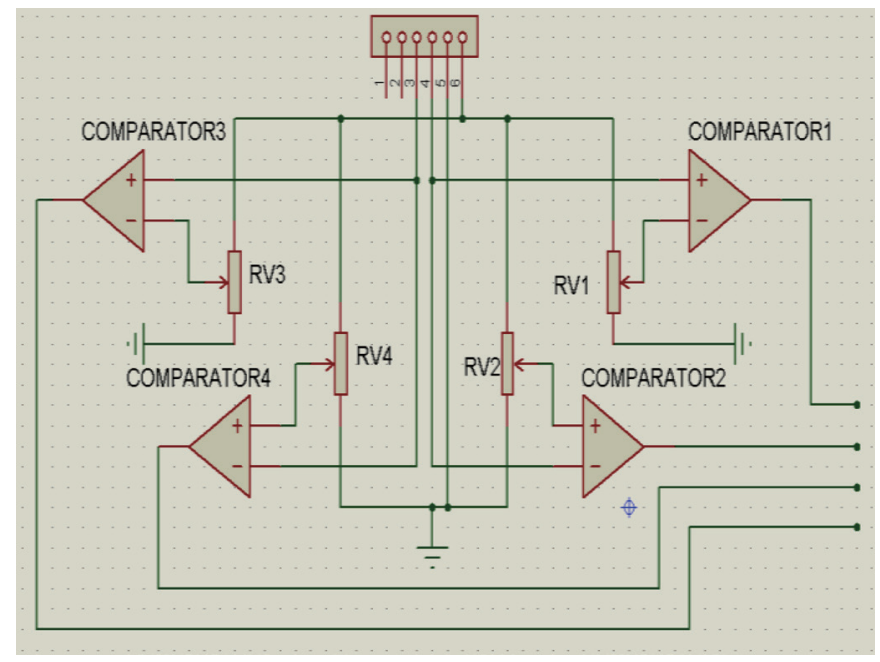

Fig. 9. (Color online) Simulation of connecting an accumulator to the voltage comparator.

\subsection{Simulation of connecting wireless RF transmitter and receiver module}

The transmitter receives the data from the HT12E encoder unit in the form of serial data in the first circuit and sends them to a similar electronic receiver in the other circuit as illustrated in Fig. 10.

\subsection{Simulation of AT89C51 controller}

This controller receives the data from the decoding unit, which will be input through its control ports for processing and sends it to the other ATMega328-PU controller as illustrated in Fig. 11.

\subsection{Simulation of connecting distance sensor to microcontroller}

In the proposed approach, we connect the pin 3 output to one of the analogue input ports of the microcontroller (ATMega328P-PU) to avoid the analogue output problem. The analog of inputl is used to connect with the sensor. Figure 12 shows the simulation of connecting the Sharp2Y0A21 sensor to the microcontroller.

\section{Results and Discussion}

The effectiveness of the SWWCHG system is validated through extensive simulations. In this section, we show the performance metrics and simulation results and analysis. 


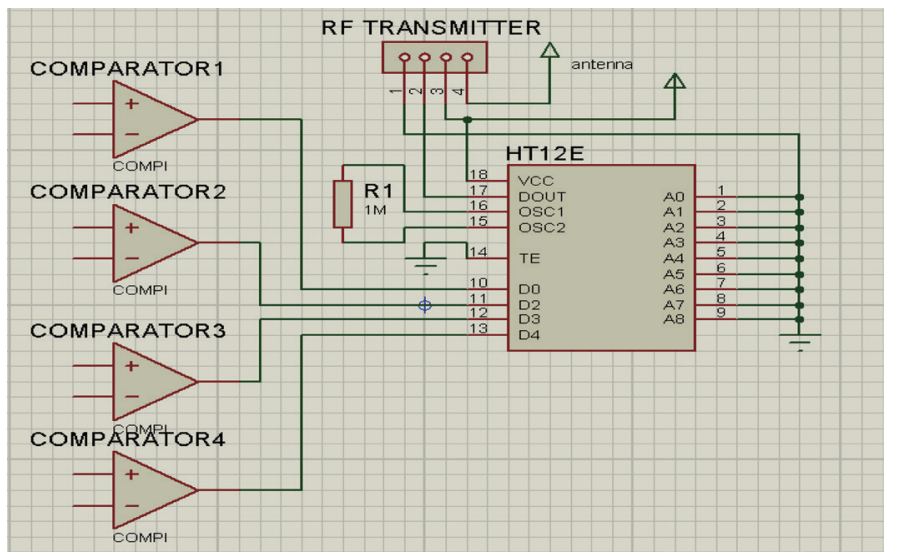

Fig. 10. (Color online) Simulation of connecting the transmitter unit with the voltage comparator and encoder unit (transmitter circuit).

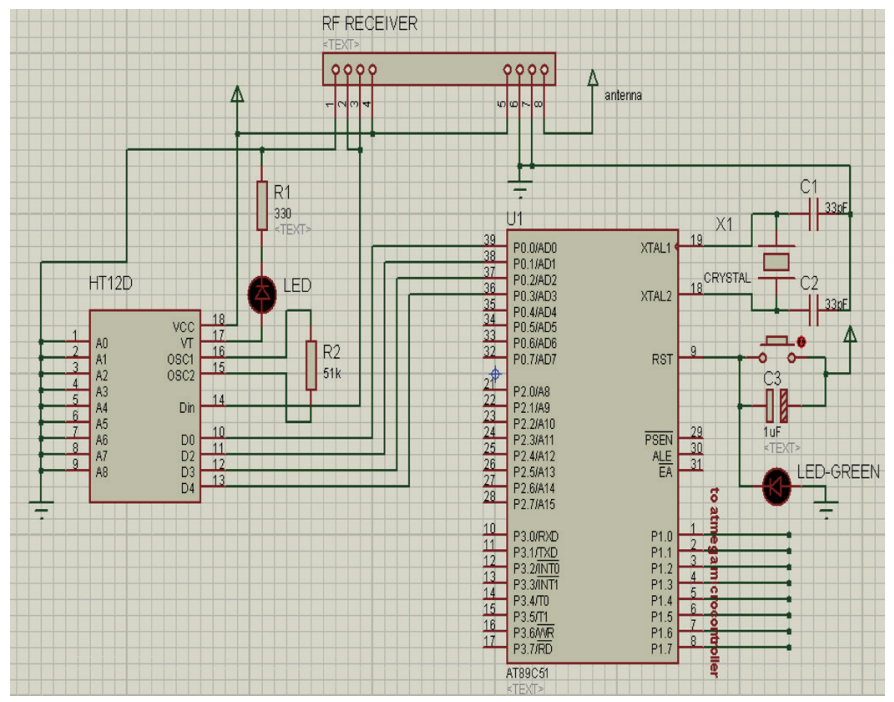

Fig. 11. (Color online) Simulation of connecting the wireless receiver unit with the decoder and AT89C51 controller (receiver circuit).

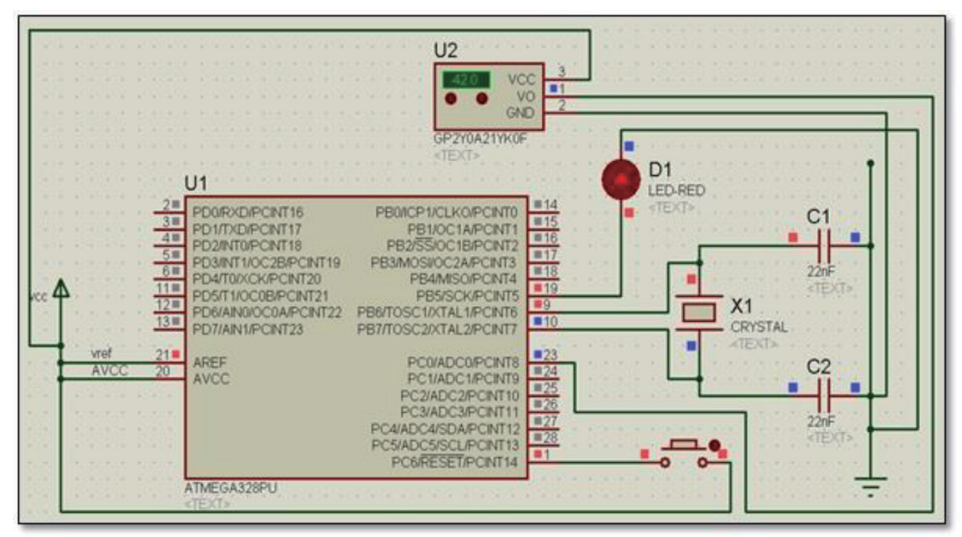

Fig. 12. (Color online) Simulation of connecting Sharp2Y0A21 sensor to microcontroller. 


\subsection{Performance metrics}

The performance of the SWWCHG system is evaluated using the following metrics:

1) hand movement change of wheelchair direction,

2) response accuracy of control commands,

3) response time of control commands,

4) issuance of distress alert if needed,

5) edge detection and avoidance, and

6) obstacle detection.

\subsection{Baseline approach}

The performance of the SWWCHG system is compared with that of the wireless smart mind wheelchair control WSMWC system. The WSMWC system involves a mind-signal and eyeblink-based control of a wheelchair. A comparison is carried out under all performance metrics explained in Sect. 5.1. The baseline approach and its objectives are stated in Table 4.

\subsection{Analysis of results}

All the components of the proposed system after integration, simulation, implementation, and experiments show that the wheelchair model works perfectly with good performance and efficiency according to the hand gestures. Table 5 shows the reaction time obtained from several experiments and trial runs of a wheelchair. The results are calculated using Eq. (1).

$$
S R=(S T \times 100) / T o T,
$$

where $S R$ is the success rate, $S T$ is the number of successful trials of wheelchair runs, and $T o T$ is the total number of trials of a wheelchair run.

The results in Table 4 show high response accuracy and rapid response. These features give high user confidence and prove the wireless control efficiency of the proposed system. The

Table 4

Baseline approach.

\begin{tabular}{lccc}
\hline Approach & Mechanism & Control nature & Objective \\
\hline WSMWC & Mind signal and eye blinks & Wireless control & Wheelchair control \\
\hline
\end{tabular}

Table 5

Success rates of the various features of the hand-gesture-controlled wheelchair.

\begin{tabular}{lc}
\hline Parameter & Success rate (\%) \\
\hline Hand movement change & 98.5 \\
Response accuracy & 93 \\
Response time & 97 \\
Distress alert & 99 \\
Edge detection and avoidance & 100 \\
Obstacle detection & 100 \\
\hline
\end{tabular}




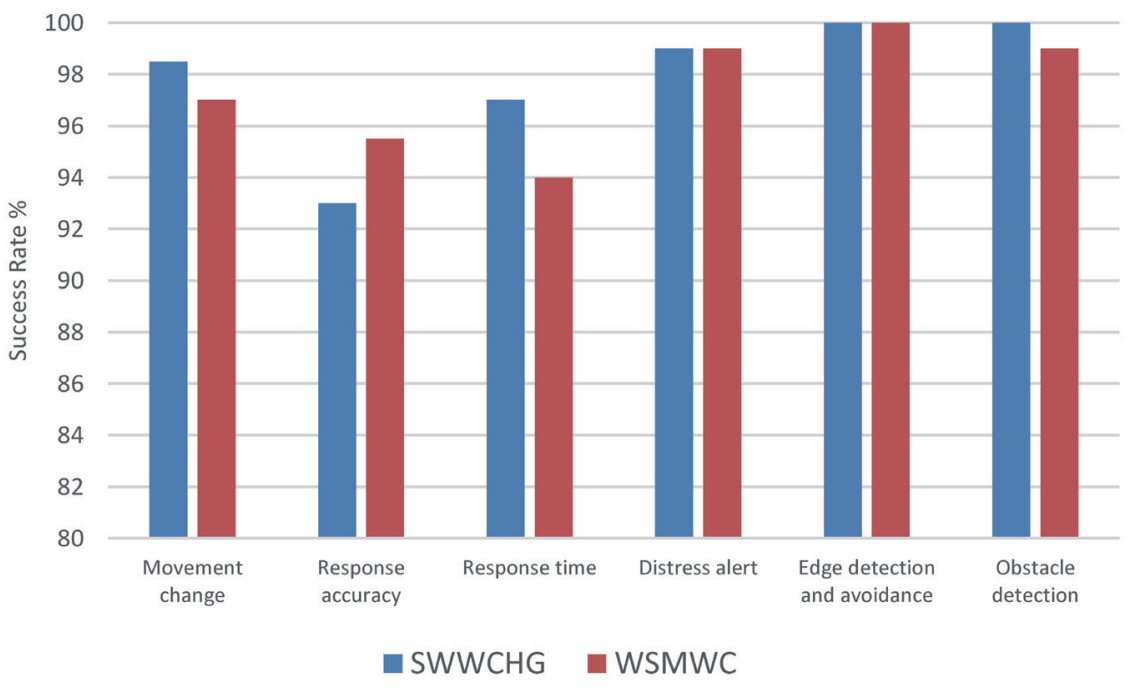

Fig. 13. (Color online) Comparative analysis of SWWCHG and WSMWC systems.

results show the capabilities of the proposed system to detect edges and obstacles, and to avoid them at a distance of $20 \mathrm{~cm}$ from the wheelchair. The results also show that approximately $1 \%$ simulation of the wheelchair turns go to other directions in the case of turns to backward as no accurate movement representation of this gesture.

\subsection{Comparative results}

Figure 13 shows a comparison of the SWWCHG and WSMWC systems under all scenarios of performance metrics. As shown in Fig. 13, the SWWCHG system outperforms the WSMWC system in terms of movement change, response time, and obstacle detection. However, the WSMWC system outperforms the SWWCHG system in terms of response accuracy because the hand gestures of people with quadriplegia are not accurate. This means that the SWWCHG system is highly reliable; thus, it is recommended and applicable to use in all types of disability.

\section{Conclusions}

The proposed system can be successfully applied on a large scale to the elderly and partially disabled people. In our proposed system, wireless technologies and sensors with relevant materials and technologies have been integrated with microcontrollers used to enhance the confidence, function, and willpower of the elderly and physically challenged people as it will help them to be self-reliant with no need to use any extra devices. The results of the proposed system show its cost-effectiveness and prove its very competitive performance, accuracy, and efficiency.

The SWWCHG system was simulated and implemented using the C programming language, Arduino C IDE, and Protues simulation software. The simulation results of the SWWCHG 
system and comparison with the WSMWC system show that the SWWCHG system is familiar to elderly and disabled people and cost-effective. Moreover, it provides very competitive performance, accuracy, and efficiency. Although the SWWCHG system is efficient, its response accuracy needs to be improved. For our future work, we will consider response accuracy and response time for all types of disability.

\section{Conflicts of Interest}

The authors declare that there are no conflicts of interest.

\section{Acknowledgments}

The authors extend their appreciation to the Deanship of Scientific Research at King Khalid University for funding this work through the General Research Project under grant number $(282 / 2019)$.

\section{References}

1 A. S. Magar and M. R. Bachute: Int. J. Eng. Sci. Comput. 6 (2016) 1356. https://doi.org/10.21474/IJAR01/287

2 G. Shreedeep, M. Somsubhra, and C. Soumya: Int. J. Elect. Electron. Data Commun. 1 (2013) 40. https://pdfs.semanticscholar.org/c507/547d83303ec9b6e2f3292c8fe40974068ad8.pdf? $\mathrm{ga}=2.169947104 .1725698896 .1564334491-1152968641.1564065420$

3 S. Magar and M. R. Bachute: Int. J. Adv. Res. 6 (2016) 1356. https://doi.org/10.21474/IJAR01/287

4 B. Abirami, S. R. Anupriya, R. Divyarani, and Nagaraju: Int. J. Technol. Res. Appl. 4 (2016) 188. https://pdfs.semanticscholar.org/252b/2c9e1525b03dc9e3056863d0ffa0cd769d26.pdf? ga $=2.174261218 .1725698896 .1564334491-1152968641.1564065420$

5 R. Shaari, M. N. Jani, and S. Ahmad: J. Eng. Sci. Res. 2 (2017) 209. https://doi.org/10.26666/rmp.jesr.2017.2.31

6 P. Gupta, P. Sharma, K. Rahul, and S. Yadav: Imperial J. Interdiscip. Res. 2 (2016) 944. http://www. imperialjournals.com/index.php/IJIR/article/view/2629/2526

7 M. Yuvaraju and R. Priyanka: Int. J. Eng. Sci. Res. Technol. 6 (2017) 543. https://zenodo.org/record/345697\#. XT8kfugzbic

8 D. Singh, V. Verma, P. Kumari, and A. Swami: Int. J. Innovations Eng. Technol. 9 (2018) 115. http://dx.doi. org/10.21172/ijiet.93.18

9 K. Sujana and N. Gunasekhar: Int. J. Sci. Eng. Technol. Res. 5 (2016) 6117. http://ijsetr.com/ uploads/546213IJSETR11360-1077.pdf

10 M. Nita, A. Kachare, and P. Lengare: Int. J. Electron. Electr. Comput. Syst. 6 (2017) 1. http://academicscience. co.in/admin/resources/project/paper/f201711121510499924.pdf

11 K. Patel, S. Patel, and M. Mansuri: Int. J. Innovative Emerg. Res. Eng. 2 (2015) 110. http://www.ijiere.com/ FinalPaper/FinalPaper20154642754249.pdf

12 R. Madane, A. Raksha, G. Radha, and T. Priyanka: Int. J. Eng. Res. Technol. 4 (2015) 131. https://doi. org/10.17577/IJERTV4IS040261

13 K. Soni, P. Vidhata, S. Yogita, and S. Pratima: Int. J. Adv. Res. Comput. Comm. Eng. 5 (2016) 344. https://doi. org/10.17148/IJARCCE.2016.5383

14 V. Pande, S. Ubale, P. Darshana, R. Nikita, and P. Pragati: J. Eng. Res. Appl. 4 (2014) 152. https://ijera.com/ papers/Vol4_issue4/Version\%204/Y04404152158.pdf

15 J. Pushpendra: Int. J. Sci. Tech. Res. 5 (2016) 18.

16 Sandeep and Supriya: Int. J. Adv. Res. Comput. Eng. Technol. 4 (2015) 1802. https://www.researchgate.net/ publication/316551968_Hand_Gesture_Controlled_Wheelchair

17 S. Salunkhe, I. Manohar, K. Kailas, and K. Shankar: Int. J. Res. Eng. Appl. Manage. 3 (2017) 7. https://www. ijream.org/papers/IJREAMV03I022636.pdf

18 M. Manda, B. Shankar, M. Abhishek, and J. Srikanth: J. Emerging Technol. Innovative Res. 5 (2018) 168. 
19 D. Goyaland and P. Saini: Int. J. Eng. Technol. 4 (2013) 15. https://www.researchtrend.net/ijet41/3\%20 DIKSHA\%20NGF.pdf

20 P. Jia, H. Hu, T. Lu, and K. Yuan: Emerald Group Publishing Limited 34 (2007) 60. https://doi. org/10.1108/01439910710718469

21 R. Xie, X. Xia, and J. Cao: IEEE Sens. J. 15 (2015) 3475. https://doi.org/10.1109/JSEN.2015.2392091

22 S. Shinde and S. Lokhande: ARIJEET 6 (2015) 1. http://v3i2.ardigitech.in/HAND\%20GESTURE\%20 CONTROLLED\%20APPLICATION\%20FOR\%20HEAVY\%20LIFTS\%20AND\%20CRANES.pdf

23 J. Francis and B. Anoop: Int. J. Comput. Appl. 99 (2014) 50. https://doi.org/10.5120/17389-7931

24 D. Goya land: Int. J. Eng. Technol. 5 (2013) 15.

25 D. Patil and B. D. Varalakshmi: Int. J. Soft Comput. Eng. 4 (2014) 45. https://pdfs.semanticscholar.org/ fa4f/52d28a1ba49ca2667452ce3d0427e084de29.pdf

\section{About the Authors}

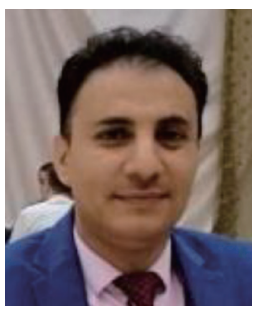

Dr. Fahd N. Al-Wesabi was born in Yemen in 1980. He received his B.S. degree in Computer Science from the University of Science and Technology, Sana'a, Yemen, in 2006, his M.S. degree in Computer Information Systems from The Arabic Academy for Banking and Financial Sciences, Sana'a branch, Yemen, in 2009, and his Ph.D. degree in Computer Science from SRTM University, India, in 2015. From 2006 to 2009, he was a research assistant, and from 2010 to 2015, he was lecturer at the Faculty of Engineering, University of Science and Technology. From 2015 to 2018, he was an assistant professor at the Faculty of Computer and Information Technology, Sana'a University, Yemen, and since October 2018, he has been an assistant professor at the Computer Science Department, King Khalid University, KSA. He is the author of eight books and more than 12 articles, and he has many funded research projects. His research interests include software engineering, applied soft computing, biosensors and simulations, soft computing tools, information security, and enterprise systems. (fwesabi@gmail.com)

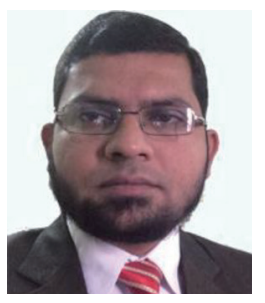

Dr. Mohammad Alamgeer was born on January 1, 1980 in Madhepur, a small village of Madhubani district in Bihar (India). He received his first religious education in Madarsa Darul-Olum, Madhepur. After some time, owing to keen interest, he was admitted to a private convent school (National Children's Academy) and after his seventh grade, he entered the government funded Jawaher High School, Madhepur. He did matriculation with 1st division in 1994 and he passed the intermediate in 1996 in science with biology as his main subject. Then, he completed his B.Sc. (Zoology Hons.) degree in 1999. Dr. Alamgeer holds an M.Sc. degree in Information Technology, an M.Sc. degree in Bioinformatics and a Master degree in Business Administration (IT). He also holds a Ph.D. degree in Bioinformatics from India. To date, he has published 13 articles in refereed journals. (dralamgeer@az-group.org) 


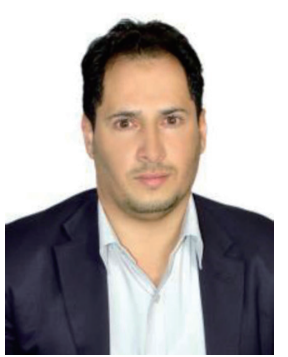

Dr. Fuad Ali Al-Yarimi was born in Yemen in November 1978. He received his B.S. degree in Computer Science from Sana'a University, Sana'a, Yemen, in 2002, his M.S. degree in Computer Information Systems from The Arabic Academy for Banking and Financial Sciences, Sana'a branch, Yemen, in 2007, and his Ph.D. degree in Computer Science from Jawaharlal Nehru University, New Delhi, India, 2014. From 2003 to 2007, he was a research assistant, and from 2007 to 2015, he was a lecturer at AlAndalus University, Faculty of Engineering and Information Systems. From 2014 to 2018, he was an assistant professor at the Faculty of Engineering and Information Systems, Al-Andalus University, Sana'a, Yemen. He was the Dean of Faculty of Engineering and Information Systems, Al-Andalus University in 2017-2018, and since October 2018, he has been an assistant professor at the Computer Science Department, King Khalid University, KSA. $\mathrm{He}$ is the author of six articles and has many funded research projects. His research interests include information privacy and security, data mining, web mining and artificial intelligence. (fuadalyarimi@gmail.com)

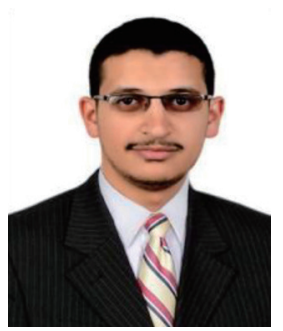

Mr. Adnan Nasr Al-Baadani was born in Yemen in 1990. He received his B.S. degree in Computer \& Network Engineering from the University of Science and Technology, Sana'a, Yemen, in 2013, he is pursuing M.S. degree in Computer Science In Sana'a University, Sana'a, Yemen. His research interests include microcontroller programming, electronics modification, and programming in general. (adnan0albaadani@gmail.com) 